\title{
Cryotherapy with liquid nitrogen versus topical salicylic acid application for cutaneous warts in primary care: randomized controlled trial
}

\author{
Sjoerd C. Bruggink MD, Jacobijn Gussekloo MD PhD, Marjolein Y. Berger MD PhD, \\ Krista Zaaijer MD, Willem J.J. Assendelft MD PhD, Margot W.M. de Waal PhD, \\ Jan Nico Bouwes Bavinck MD PhD, Bart W. Koes PhD, Just A.H. Eekhof MD PhD
}

Previously published at www.cmaj.ca

\section{ABSTRACT}

Background: Cryotherapy is widely used for the treatment of cutaneous warts in primary care. However, evidence favours salicylic acid application. We compared the effectiveness of these treatments as well as a wait-and-see approach.

Methods: Consecutive patients with new cutaneous warts were recruited in 30 primary care practices in the Netherlands between May 1, 2006, and Jan. 26, 2007. We randomly allocated eligible patients to one of three groups: cryotherapy with liquid nitrogen every two weeks, self-application of salicylic acid daily or a wait-and-see approach. The primary outcome was the proportion of participants whose warts were all cured at 13 weeks. Analysis was on an intention-totreat basis. Secondary outcomes included treatment adherence, side effects and treatment satisfaction. Research nurses assessed outcomes during home visits at 4, 13 and 26 weeks.

Results: Of the 250 participants (age 4 to 79 years), 240 were included in the analysis at 13 weeks (loss to follow-up 4\%). Cure rates were $39 \%$ (95\% confidence interval $[\mathrm{Cl}] 29 \%-51 \%$ ) in the cryotherapy group, $24 \%(95 \% \mathrm{Cl} 16 \%-35 \%)$ in the salicylic acid group and $16 \%(95 \% \mathrm{Cl} 9.5 \%-25 \%)$ in the wait-andsee group. Differences in effectiveness were most pronounced among participants with common warts $(n=116)$ : cure rates were $49 \%(95 \% \mathrm{Cl} 34 \%-64 \%)$ in the cryotherapy group, 15\% (95\% Cl 7\%-30\%) in the salicylic acid group and $8 \%(95 \% \mathrm{Cl}$ $3 \%-21 \%)$ in the wait-and-see group. Cure rates among the participants with plantar warts $(n=124)$ did not differ significantly between treatment groups.

Interpretation: For common warts, cryotherapy was the most effective therapy in primary care. For plantar warts, we found no clinically relevant difference in effectiveness between cryotherapy, topical application of salicylic acid or a wait-and-see approach after 13 weeks. (ClinicalTrial.gov registration no. ISRCTN42730629)

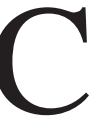
utaneous warts are common. ${ }^{1-3} \mathrm{Up}$ to one-third of primary school children have warts, of which twothirds resolve within two years. ${ }^{4.5}$ Because warts frequently result in discomfort, ${ }^{6} 2 \%$ of the general population and $6 \%$ of school-aged children each year present with warts to their family physician. ${ }^{7.8}$ The usual treatment is cryotherapy with liquid nitrogen or, less frequently, topical application of salicylic acid. ${ }^{9-12}$ Some physicians choose a wait-and-see approach because of the benign natural course of warts and the risk of side effects of treatment. ${ }^{10,11}$

A recent Cochrane review on treatments of cutaneous warts concluded that available studies were small, poorly designed or limited to dermatology outpatients. ${ }^{10,11}$ Evidence on cryotherapy was contradictory, ${ }^{13-18}$ whereas the evidence on salicylic acid was more convincing. ${ }^{19-23}$ However, studies that compared cryotherapy and salicylic acid directly showed no differences in effectiveness. ${ }^{24,25}$ The Cochrane review called for high-quality trials in primary care to compare the effects of cryotherapy, salicylic acid and placebo.

We conducted a three-arm randomized controlled trial to compare the effectiveness of cryotherapy with liquid nitrogen, topical application of salicylic acid and a wait-and-see approach for the treatment of common and plantar warts in primary care.

\section{Methods}

\section{Participants}

Between May 1, 2006, and Jan. 26, 2007, 30 family practices from the Leiden Primary Care Research Network in the Netherlands invited all patients aged four years and older who attended the clinic with one or more new cutaneous warts to participate. We defined new cutaneous warts as those on the skin that were diagnosed in family practice and had not been treated by a physician or dermatologist in the previous year, regardless of previous self-treatment with over-the-counter medication. We excluded immunocompromised patients and patients with genital warts, seborrheic warts or warts larger than $1 \mathrm{~cm}$ in diameter. Patients who fulfilled the inclusion cri-

From the Department of Public Health and Primary Care (Bruggink, Gussekloo, Zaaijer, Assendelft, de Waal, Eekhof); the Department of Dermatology (Bouwes Bavinck), Leiden University Medical Center, Leiden, the Netherlands; and the Department of General Practice (Berger, Koes), Erasmus University Medical Center, Rotterdam, the Netherlands

CMAJ 2010. DOI:10.1503/cmaj.092194 
teria and agreed to participate were visited at home by a trained research nurse, who confirmed their eligibility. Informed consent (child as well as parental informed consent for participants less than 18 years of age) was obtained, and baseline characteristics were collected.

\section{Study design and randomization}

We stratified patients by location of warts: plantar (warts on the soles of the feet) or common (warts on the hands or other locations). ${ }^{26}$ Participants who had both plantar and common warts were stratified according to where the majority of their warts were located. We used opaque, sealed envelopes that were numbered based on a computerized randomization list delivered by an independent statistician to conceal allocation. After stratification by location of warts and by number of warts (< six warts v. $\geq$ six warts), random allocation of participants to treatment groups was done without blocking. The study protocol was approved by the medical ethical committee of the Leiden University Medical Center.

\section{Treatment protocols}

One of us (K.Z.) trained all participating family physicians and assistants working in their practices in the three 13-week treatment protocols, which were designed to reflect best practice. ${ }^{10,24}$ Training consisted of a one-hour interactive practical session, during which all tools and techniques were demonstrated; real warts were not used in the demonstrations.

For cryotherapy, we used a high-intensity regimen of one session every two weeks until all warts were completely gone. During each session, the participant received three serial applications in which a wad of cotton wool saturated with liquid nitrogen was moved around on the wart. Each application was executed until a frozen halo of $2 \mathrm{~mm}$ around the base of the wart appeared (usually after 2-10 seconds).

For the topical application of salicylic acid, we used a white petroleum jelly containing $40 \%$ salicylic acid. We chose this concentration to provide a stronger treatment than over-the-counter products, which usually contain $17 \%$ salicylic acid. Participants assigned to this group were asked to apply the salicylic acid every day until the warts were completely gone. They were instructed to cover the surrounding skin with tape to protect healthy skin and apply the salicylic acid on top of the wart with another piece of tape. Before each subsequent daily application, they used a file to pare the softened surface area of the wart.

Participants assigned to the wait-and-see group were informed about the benign natural course of warts and were advised not to undergo treatment (apart from over-the-counter medication) for at least 13 weeks.

After the 13-week treatment period, all participants who still had warts could switch to another treatment according to their own preferences. Participants were free to use over-thecounter medication during the entire follow-up period but were asked to report all usage.

\section{Outcome measures}

Trained research nurses assessed outcomes during home visits at 4, 13 and 26 weeks of follow-up, independently of the treating physicians. A wart was considered cured if it was no longer visible (skin colour and skin lines were reestablished) and could not be palpated anymore by hand. The primary outcome measure was the proportion of participants whose warts were all cured at 13 weeks. Research nurses assessed side effects, newly developed warts (which were not included in the primary outcome assessment) and adherence to treatment. Treatment adherence was considered adequate if participants had received cryotherapy at least every three weeks, had self-administered salicylic acid at least four days per week and had not undergone any cointervention (treatment of warts other than over-the-counter medication).

In addition, participants were asked to rate treatment burden using a 10-point scale $(1=$ no burden, $10=$ the worst imaginable burden). A scores of six or higher was considered to reflect a substantial burden. Participants rated treatment satisfaction using a five-point scale (one = very unsatisfied, five $=$ very satisfied); those with a score of four or five were considered to be satisfied.

Research nurses, family physicians and participants were not blinded to treatment allocation. For quality control, 5\% of the assessments were directly supervised by experienced family physicians (J.E. and K.Z.).

\section{Statistical analysis}

We chose a sample size that would provide $80 \%$ power, at a significance level of $5 \%$, to detect an absolute increase in the cure rate of $20 \%$ between the two active treatment groups. Based on a literature review, we expected salicylic acid to be most effective, with a $70 \%$ cure rate. ${ }^{10,11}$ A total of 91 patients were required per treatment arm.

We used the $\chi^{2}$ test for all comparisons of cure rates and percentages. In our primary analysis, we compared cure rates between the three treatment arms on an intention-to-treat basis. We also calculated relative risks, risk differences and numbers needed to treat for cryotherapy versus salicylic acid, cryotherapy versus wait-and-see approach, and salicylic acid versus wait-and-see approach.

In secondary analyses, we compared cure rates between the three study arms (a) with patients lost to follow-up considered not cured, (b) after excluding patients who had both plantar and common warts, (c) at 26 weeks' follow-up, (d) using individual warts as the unit of analysis instead of patients and (e) per protocol cure rates based on reported treatment adherence.

Subgroup analyses were pre-planned for location of warts (common wart group v. plantar wart group), age clusters (412 years v. $\geq 12$ years), number of warts per participant, and duration of warts ( $\leq$ six months $v$. $>$ six months). We formally tested for effect modification of treatment by location of warts using a logistic regression model.

Lastly, we compared the percentages of patients with side effects and considerable treatment burden between the two active treatment arms, and the percentages of patients satisfied with treatment between the three arms.

An abridged version of our study protocol can be found at www.controlled-trials.com/ISRCTN42730629/warts. 


\section{Results}

\section{Patient characteristics}

Of 303 patients recruited, we excluded 53, mainly because they had already received treatment in the previous year or refused to participate (Figure 1). We randomly assigned the remaining 250 participants to the cryotherapy ( $n=80)$, topical salicylic acid $(n=84)$ and wait-and-see $(n=86)$ groups. Baseline characteristics did not differ significantly between the groups (Table 1). Seven per cent of the participants reported that they had received treatment for warts more than one year before enrolment; $35 \%$ reported that they had

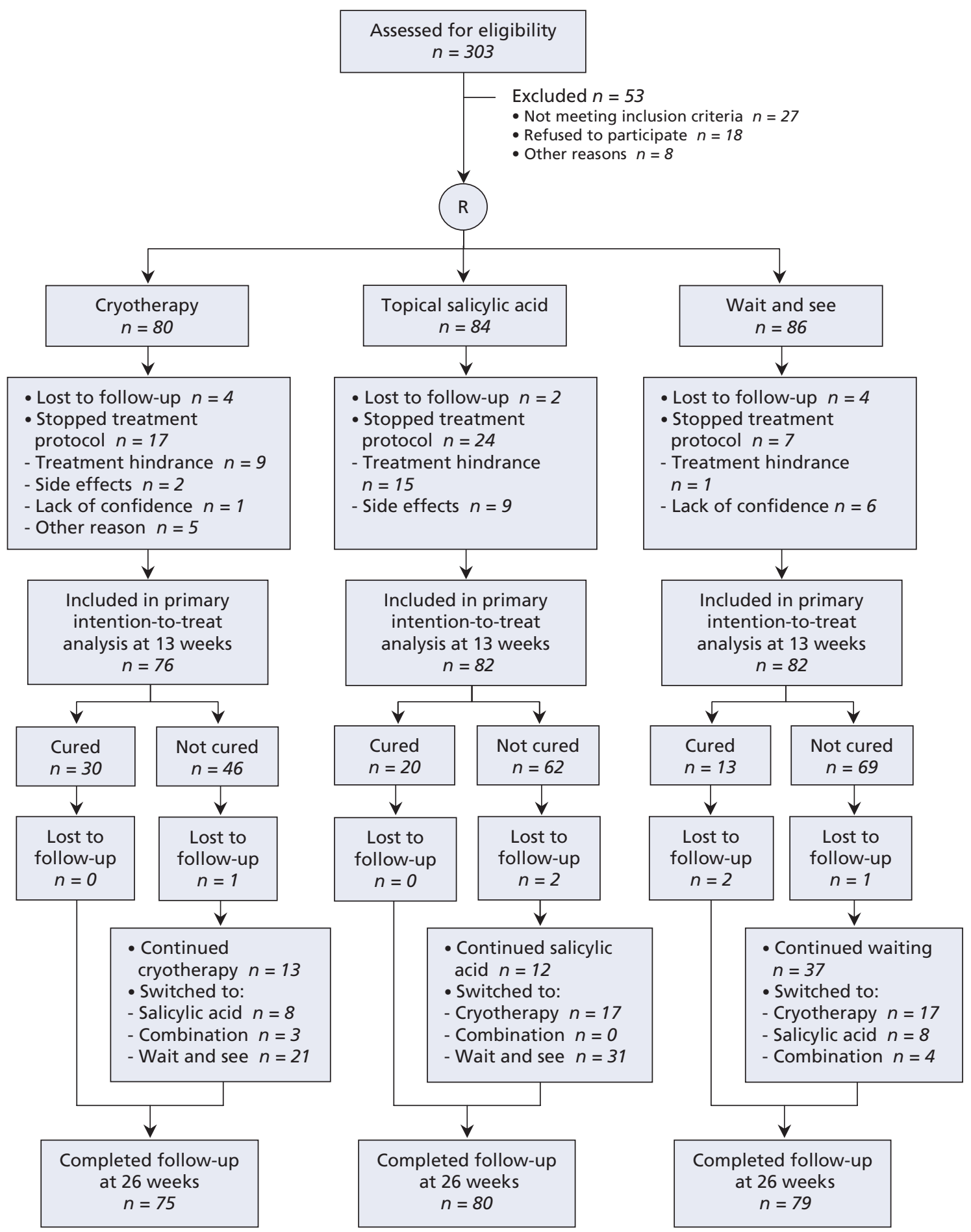

Figure 1: Selection of patients for the study. 
treated their warts with one or more of the following overthe-counter medications or methods in the past with no success: dimethylether propane cryotherapy (18\%), ointment containing salicylic acid at a concentration lower than the study ointment (12\%), cutting away the warts themselves $(6 \%)$ and other alternatives $(6 \%)$. At study entry, $34 \%$ of the participants stated that they preferred cryotherapy, 35\% salicylic acid and $4 \%$ a wait-and-see approach (no preference given by $27 \%$ ).

Of the 250 participants, $122(49 \%)$ were stratified into the common wart group and $128(51 \%)$ into the plantar wart group. In the common wart group, 103 participants (84\%) had warts on their hands, 19 (16\%) had them on parts of the body other than hands or soles of the feet, and $13(11 \%)$ also had plantar warts. In the plantar wart group, 22 participants (17\%) also had common warts. Baseline characteristics were similar between the common and plantar wart groups except for age distribution and duration of warts.

\section{Follow-up and treatment adherence}

At 13 weeks, 10 participants (4\%) were lost to follow up (8 refused further participation, 1 had entered by error because the wart was diagnosed as a seborrheic wart, and 1 was lost for unknown reasons). Overall, 48 (20\%) of the remaining 240 participants stopped the assigned treatment protocol (see Appendix 1, available at www.cmaj.ca/cgi/content/full/cmaj
$.092194 / \mathrm{DC} 1)$. During the 13-week follow-up period, 61 participants $(25 \%)$ had one or more new warts; no participants were referred to dermatology outpatient clinics.

\section{Effectiveness of treatment}

At 13 weeks, the cure rates were $39 \%$ (95\% confidence interval [CI] 29\%-51\%) after cryotherapy, 24\% (95\% CI 16\%$35 \%)$ after salicylic acid and 16\% (95\% CI 9.5\%-25\%) after the wait-and-see protocol, for a relative risk of $1.6(95 \% \mathrm{CI}$ 1.0-2.6) for cryotherapy versus salicylic acid. Because the effectiveness of treatments differed between the common wart group and the plantar wart group ( $p$ for interaction 0.007), we report outcomes for all patients as well as by location of warts (Tables 2 and 3).

In the common wart group, cryotherapy was most effective, with a cure rate of $49 \%(95 \%$ CI $34 \%-64 \%)$ at 13 weeks (Tables 2 and 3). Further stratification by age and by duration of warts gave similar findings.

In the plantar wart group, the cure rate at 13 weeks did not differ between the treatment arms (Tables 2 and 3). Further stratification revealed that cure rates were considerably lower among participants 12 years and older than among younger participants. Also, cure rates were lower among participants whose warts had been present for six or more months at baseline than among those whose warts had been present for a shorter duration (Table 2).

Table 1: Baseline characteristics of 250 patients with cutaneous warts randomly assigned to one of three treatment arms

\begin{tabular}{|c|c|c|c|}
\hline \multirow[b]{2}{*}{ Characteristic } & \multicolumn{3}{|c|}{ Treatment arm; no. (\%) of participants* } \\
\hline & $\begin{array}{c}\text { Cryotherapy } \\
n=80\end{array}$ & $\begin{array}{l}\text { Topical salicylic acid } \\
\qquad n=84\end{array}$ & $\begin{array}{l}\text { Wait-and-see approach } \\
\qquad n=86\end{array}$ \\
\hline Sex, female & $45(56)$ & $54(64)$ & $50(58)$ \\
\hline \multicolumn{4}{|l|}{ Age, yr } \\
\hline $4-12$ & $33(41)$ & $36(43)$ & $39(45)$ \\
\hline$\geq 12$ & $47(59)$ & $48(57)$ & $47(55)$ \\
\hline No. of warts, median (IQR) & $2(1-3)$ & $2(1-4)$ & $2(1-5)$ \\
\hline Size of warts, mm, median (IQR) & $4(3-5)$ & $4(3-5)$ & $4(3-5)$ \\
\hline Hindrancet & $55(69)$ & $63(75)$ & $70(81)$ \\
\hline \multicolumn{4}{|l|}{ Location of warts $\ddagger$} \\
\hline Plantar (soles of the feet) & $39(49)$ & $44(52)$ & $45(52)$ \\
\hline Common (hands or other) & $41(51)$ & $40(48)$ & $41(48)$ \\
\hline \multicolumn{4}{|l|}{ Duration of warts, mo } \\
\hline$<6$ & $31(39)$ & $37(44)$ & $34(40)$ \\
\hline$\geq 6$ & $49(61)$ & $47(56)$ & $52(60)$ \\
\hline \multicolumn{4}{|l|}{ Treatment preference at baseline } \\
\hline Cryotherapy & $33(41)$ & $24(29)$ & $29(34)$ \\
\hline Salicylic acid & $22(28)$ & 33 (39) & $32(37)$ \\
\hline Wait-and-see approach & $6(8)$ & $2(2)$ & $2(2)$ \\
\hline No preference & $19(24) \S$ & $25(30)$ & $23(27)$ \\
\hline
\end{tabular}

Note: IQR = Interquartile range

*Unless stated otherwise.

tPresence of pain, irritation or esthetic annoyance of warts.

¥Number of participants with common warts and number of participants with plantar warts were equivalent by chance.

§Sum of percentages is not equal to 100 because of rounding 
Table 2: Effectiveness of treatments at 13 weeks, by location of warts $(n=240)$

Treatment arm; no. (\%) of participants cured*

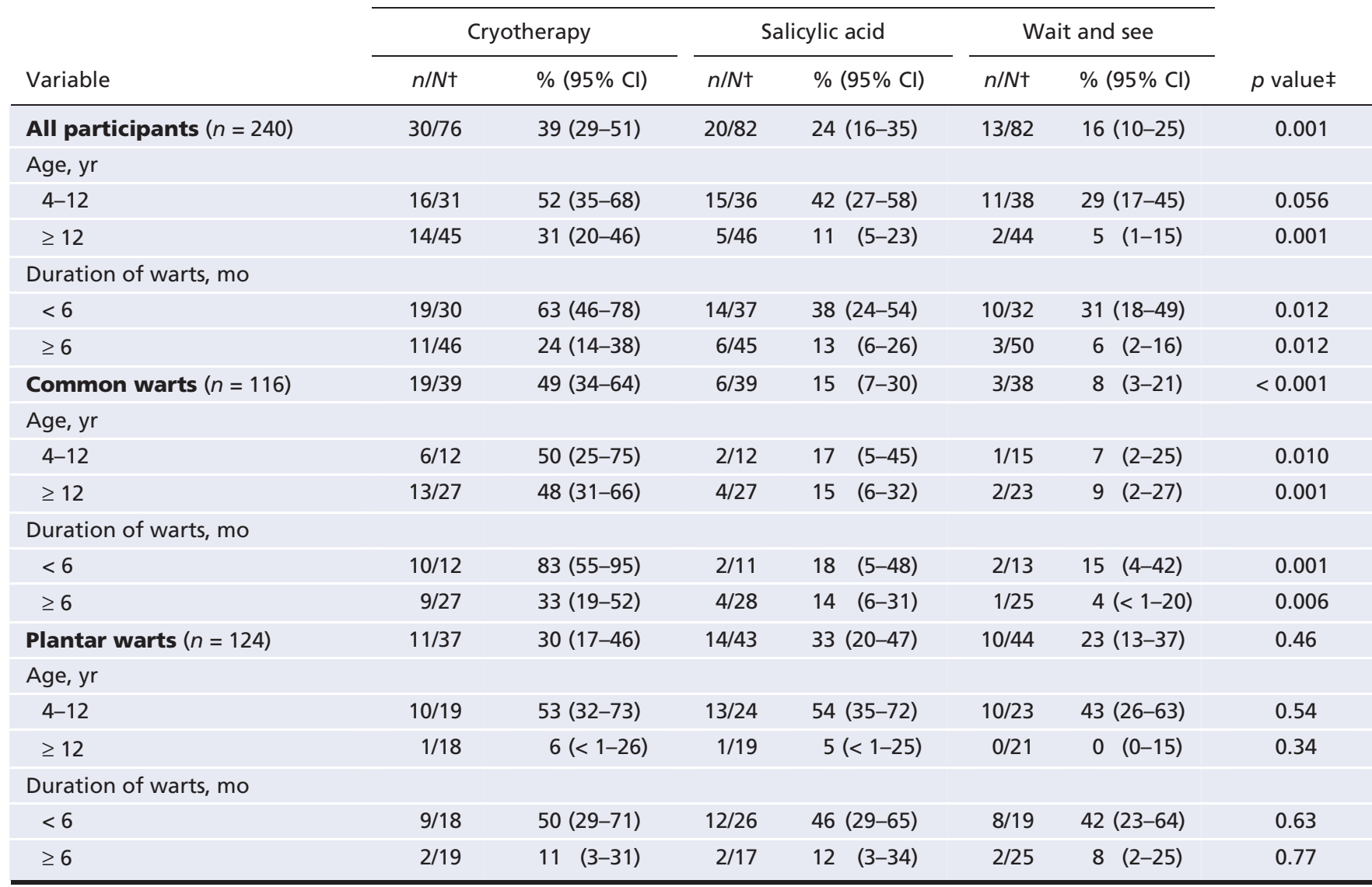

Note: $\mathrm{Cl}=$ confidence interval.

*A participant was considered cured when all warts present at baseline were gone at follow-up.

tNumber of participants cured / number of participants in intention-to-treat analysis at 13 weeks, and percentages of participants cured.

$\ddagger$ Two-sided $\chi^{2}$, linear-by-linear association, comparing three treatment groups.

Table 3: Relative measures of effect between the three treatment arms at 13 weeks, by location of warts $(n=240)$

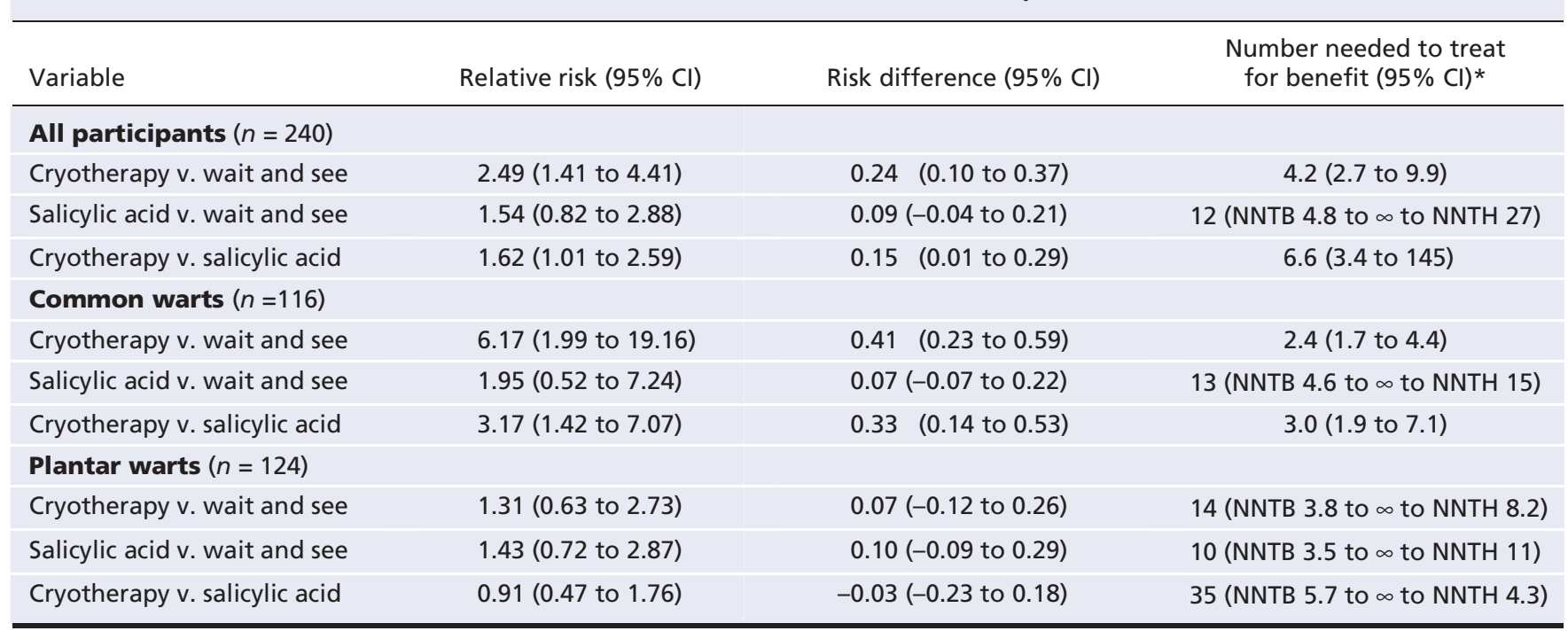

Note: $\mathrm{Cl}=$ confidence interval, NNTB = number needed to treat for benefit, NNTH = number needed treat for harm.

*Where the $95 \% \mathrm{Cl}$ of the risk difference overlaps zero, the corresponding $95 \% \mathrm{Cl}$ of the number needed to treat includes infinity ( $\infty$ ) and therefore is expressed as a range from NNTB(benefit) to $\infty$ to NNTH(harm). 


\section{Sensitivity analysis}

The results at 26 weeks were concordant with the results at 13 weeks (see Appendix 2, available at www.cmaj.ca/cgi/content /full/cmaj.092194/DC1). The same was true when we considered that all patients lost to follow-up were not cured, or when we excluded participants with both common and plantar warts from the analysis. Per-protocol analysis and analysis of the cure rate of individual warts at 13 weeks showed the same significant results as our primary analysis (see Appendices 3 and 4, available at www.cmaj.ca/cgi/content/full/cmaj.092194/DC1) .

\section{Side effects and treatment satisfaction}

In both wart groups, participants experienced more side effects after cryotherapy than after topical salicylic acid application (see Appendix 5, available at www.cmaj.ca/cgi/content /full/cmaj.092194/DC1). The side effects included pain, blistering, scarring, skin irritation, skin pigmentation and crust. In the common wart group, $31 \%$ (95\% CI 19\%-46\%) of the participants reported considerable treatment burden after cryotherapy and 54\% (95\% CI 39\%-68\%) after salicylic acid treatment $(p=0.040)$. Furthermore, 69\% (95\% CI 53\%-82\%) of participants were satisfied with treatment after cryotherapy, as compared with $24 \%(95 \%$ CI $13 \%-39 \%)$ after salicylic acid treatment and 22\% (95\% CI 12\%-38\%) after the waitand-see protocol $(p<0.001)$. In the plantar wart group, there were no differences in treatment burden or satisfaction between the three treatment groups.

\section{Interpretation}

In this pragmatic three-arm randomized controlled trial conducted in family practices, we found that cryotherapy was the most effective therapy for common warts (mainly on hands), with $49 \%$ of patients cured after 13 weeks. Despite the fact that cryotherapy caused more frequent and more severe side effects than topical salicylic acid application, patients were most satisfied when treated with cryotherapy. For plantar warts, we found no clinically relevant difference between the treatment arms. Regardless of treatment, children with plantar warts showed relatively high cure rates (about 50\%), whereas plantar warts in adolescents and adults were highly persistent (cure rates of about 5\%).

Although our overall relative risk of 1.5 between salicylic acid treatment and the wait-and-see protocol was similar to the relative risk of 1.6 from pooled data in the recent Cochrane review, our overall cure rates of $24 \%$ in the salicylic acid group and $16 \%$ in the wait-and-see group were lower than the cure rates of $73 \%$ and $48 \%$ in the Cochrane review at similar followup. ${ }^{10,11}$ This marked difference is most likely due to variation in study design and study population. Our primary care setting, pragmatic design, wide inclusion criteria, excellent follow-up and intention-to-treat analysis led to results that were easy to interpret and directly applicable to daily practice in primary care. In contrast, the two other studies comparing cryotherapy and salicylic acid treatment, which involved dermatology outpatients, excluded patients who had more than five warts, those with warts on locations other than the location under investigation, and nonattending or noncompliant patients (in our study
$20 \%$ of participants included in the analysis were noncompliant). ${ }^{24,25}$ Other factors may also be at play, such as age of the patients and duration of warts before treatment, which our study showed to be significantly associated with cure rates.

Our follow-up at 26 weeks showed that the effects of treatment of common warts were sustainable. In the plantar wart group, in contrast to statistically equal effectiveness at 13 weeks, both of the active treatments might have higher cure rates than a wait-and-see approach in the long term. These findings suggest that the effect of active treatments on plantar warts is delayed or that more aggressive treatment is needed because of the callosity overlying the warts. ${ }^{14}$

\section{Limitations}

As in daily practice, salicylic acid was applied by the participants themselves, which could reduce effectiveness compared with treatments applied by health professionals. However, we explicitly recorded participants' adherence to standardized treatment protocols, and intention-to-treat cure rates were concordant with results of the per-protocol analyses.

The participating patients and family practices were aware of the treatment allocations, because the pragmatic study design and treatment options did not secure realistic blinding. Furthermore, the research nurses who assessed outcomes were aware of the treatment allocations, because the appearance of the skin after treatment usually revealed the specific treatment and because the large proportion of children often spontaneously reported the specific treatment.

\section{Conclusion}

Although earlier evidence favoured topical salicylic acid application over cryotherapy for the treatment of cutaneous warts, the results of our randomized controlled trial provides evidence to support the use of cryotherapy over salicylic acid treatment, for common warts only. For plantar warts, we found no clinically relevant difference between cryotherapy, salicylic acid treatment or a wait-and-see approach after 13 weeks.

This article has been peer reviewed.

Competing interests: None declared.

Contributors: Jacobijn Gussekloo, Marjolein Berger, Willem Assendelft and Just Eekhof were responsible for the study concept and design. Jacobijn Gussekloo, Marjolein Berger, Willem Assendelft, Jan Nico Bouwes Bavinck, Bart Koes and Just Eekhof were responsible for the original study protocol. Sjoerd Bruggink contributed to the interpretation and analysis of data and drafted the manuscript. Jacobijn Gussekloo contributed to the analysis of data. Krista Zaaijer was responsible for the recruitment of participants. Krista Zaaijer, Margot de Waal and Just Eekhof contributed to the collection of data and the interpretation or analysis of data. All of the authors revised the manuscript critically for important intellectual content and approved the final version submitted for publication. All of the authors had full access to all of the data and take responsibility for the integrity of the data and the accuracy of the analysis.

Acknowledgements: The authors thank all of the participating patients and family practices from the Leiden Primary Care Research Network as well as the trial research nurses Els de Haas-van Rijn and Carin Mostert-Westdijk.

Funding: The study was supported by the Netherlands Organisation for Health Research and Development (Fund Common Diseases). The organization did not have any influence on the study design, the collection, analysis or interpretation of data, the writing of the report or the decision to submit the paper for publication. 


\section{REFERENCES}

1. Rea JN, Newhouse ML, Halil T. Skin disease in Lambeth. A community study of prevalence and use of medical care. Br J Prev Soc Med 1976;30:107-14.

2. Johnson MT, Roberts J. Skin conditions and related need for medical care among persons 1-74 years. United States, 1971-1974. Vital Health Stat 1978;i-72.

3. Beliaeva TL. The population incidence of warts [article in Russian]. Vestn Derma tol Venerol 1990;2:55-8.

4. van Haalen FM, Bruggink SC, Gussekloo J, et al. Warts in primary school children Prevalence and relation with environmental factors. Br J Dermatol 2009;161:148-52.

5. Massing AM, Epstein WL. Natural history of warts. A two-year study. Arch Dermatol 1963;87:306-10.

6. Dudley W. The psychological impact of warts on patients' lives. Prof Nurse 1995;11:99-100.

7. Westert GP, Schellevis FG, de Bakker DH, et al. Monitoring health inequalities through general practice: the Second Dutch National Survey of General Practice. Eur J Public Health 2005;15:59-65.

8. Office of Population Censuses and Surveys. Morbidity statistics from general practice, fourth national study 1991-1992 [series MB5 no. 3]. London (UK): The Office; 1995. Available: www.statistics.gov.uk/downloads/theme_health /MB5No3.pdf (accessed 2010 Aug. 18).

9. Bruggink SC, Waagmeester SC, Gussekloo J, et al. Current choices in treatment of cutaneous warts: a survey among Dutch general practitioners. Fam Pract 2010; July 7 [Epub ahead of print]

10. Gibbs S, Harvey I. Topical treatments for cutaneous warts [review]. Cochrane Database Syst Rev 2006;(3):CD001781.

11. Thomas KS, Keogh-Brown MR, Chalmers JR, et al. Effectiveness and costeffectiveness of salicylic acid and cryotherapy for cutaneous warts. An economic decision model. Health Technol Assess 2006;10:iii, ix-87.

12. Androphy EJ, Lowy DR. Warts. In: Wolff K, Goldsmith LA, Katz SI, et al., editors. Fitzpatrick's dermatology in general medicine. 7th ed. New York (NY): McGraw-Hill; 2008. p. 1914-23.

13. Hansen JG, Schmidt H. Plantar warts. Occurrence and cryosurgical treatment [article in Danish]. Ugeskr Laeger 1986;148:173-4.

14. Sonnex TS, Camp RDR. The treatment of recalcitrant viral warts with high dose cryosurgery under local anaeshesia. Br J Dermatol 1988;119:38-9.

15. Berth-Jones J, Bourke J, Eglitis H, et al. Value of a second freeze-thaw cycle in cryotherapy of common warts. Br J Dermatol 1994;131:883-6.

16. Connolly M, Bazmi K, O'Connell M, et al. Cryotherapy of viral warts: a sustained 10-s freeze is more effective than the traditional method. Br J Dermatol 2001;145:554-7.

17. Wilson P. Immunotherapy v cryotherapy for hand warts; a controlled trial [abstract]. Scott Med J 1983;28:191.

18. Gibson JR, Harvey SG, Barth J, et al. A comparison of acyclovir cream versus placebo cream versus liquid nitrogen in the treatment of viral plantar warts. Dermatologica 1984;168:178-81.

19. Bunney MH, Hunter JA, Ogilvie MM, et al. The treatment of plantar warts in the home. A critical appraisal of a new preparation. Practitioner 1971;207:197-204

20. Flindt-Hansen H, Tikjob G, Brandrup F. Wart treatment with anthralin. Acta Derm Venereol 1984;64:177-9.

21. Auken G, Gade M, Pilgaard CE. Treatment of warts of the hands and feet with Verucid [article in Danish]. Ugeskr Laeger 1975;137:3036-8.

22. Bart BJ, Biglow J, Vance JC, et al. Salicylic acid in karaya gum patch as a treatment for verruca vulgaris. J Am Acad Dermatol 1989;20:74-6.

23. Spanos NP, Williams V, Gwynn MI. Effects of hypnotic, placebo, and salicylic acid treatments on wart regression. Psychosom Med 1990;52:109-14.

24. Bunney MH, Nolan MW, Williams DA. An assessment of methods of treating viral warts by comparative treatment trials based on a standard design. $\mathrm{Br} J \mathrm{Der}$ matol 1976;94:667-79.

25. Steele K, Irwin WG. Liquid nitrogen and salicylic/lactic acid paint in the treatment of cutaneous warts in general practice. J R Coll Gen Pract 1988;38:256-8.

26. Jablonska S, Majewski S, Obalek S, et al. Cutaneous warts. Clin Dermatol 1997;15:309-19.

Correspondence to: Dr. Sjoerd C. Bruggink, Department of Public Health and Primary Care, Leiden University Medical Center,

PO Box 9600, 2300 RC, Leiden, the Netherlands;

s.c.bruggink@lumc.nl

\section{"ACCUPRIL ${ }^{\circledR}$ and "ACCURETIC ${ }^{\circledR}$} StIll fighting
hypertension

$\checkmark$ Powerful BP-lowering ${ }^{1}$

$\checkmark$ 24-hour BP control demonstrated with ACCUPRIL ${ }^{1}$

$\checkmark$ The flexibility of seven dosage strengths with ACCUPRIL and ACCURETIC ${ }^{1,2}$

$\checkmark$ Al dosages at a single flat price ${ }^{3 \dagger}$ †Price does not include pharmacy professional fees. Please refer to Product Monographs for complete dosing information.
P

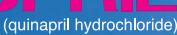

TAKE A FORWARD STEP

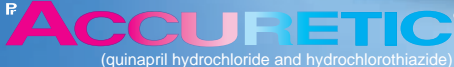

POWER OF COMBINED CONTROL
PRIL is indicated in essential hypertension when diuretics or betackers are unsuitable.

WARNINGS: As with all ACE inhibitors, please refer to specific warnings regarding drug discontinuation in angioedema and pregnancy.

The most frequent adverse events in controlled clinical trials with ACCUPRIL were headache $(8.1 \%)$, dizziness $(4.1 \%)$, cough $(3.2 \%)$, fatigue $(3.2 \%)$, $3.2 \%$ ), nausea and/or vomiting $(2.3 \%)$ and abdominal pain $(2.0 \%)$. olete list of adverse events, please refer to the Product theranv

Quin is contraindicated in pregnancy. ACE inhibitors can cause fetal ar - morbidity and mortality when administered to pregnant women. Several dozen cases have been reported in the world literature. Qu napril should not be used by women who are pregnant, intend to become pregnant, or could become pregnant and who are not using adeouate contraceptive measures. It is possible that quinapril passes into breast milk. Patients should be advised not to breast-feed while taking quinapril. See prescribing information for complete contraindications.

When used in pregnancy, angiotensin converting enzyme (ACE) inhilititors can cause injury or even death of the developing fetus. When pregnancy is detected, ACCUPRIL or ACCURETIC should be discontinued as soon as possible.

The most frequent adverse events in controlled trials with ACCURETIC were headache $(6.7 \%)$, dizziness $(4.8 \%)$, cough $(3.2 \%)$ and fatigue $(2.9 \%)$. For the complete list of adverse events, please refer to the Product Monograph. 